\title{
Anticancer effect of inactivated Sendai virus strain Tianjin on human osteosarcoma HOS cells
}

\author{
Qing $\mathrm{Li}^{1, \star}$, Huachong $\mathrm{Ma}^{2, \star}$, Shuya Sun ${ }^{1}$ and Liying $\mathrm{Shi}^{1}$ \\ ${ }^{1}$ Department of Pathogenic Biology, School of Basic Medical Sciences, Tianjin Medical University, Tianjin, China \\ ${ }^{2}$ Department of General Surgery, Beijing Chaoyang Hospital, Capital Medical University, Beijing, China
}

\begin{abstract}
Ultraviolet-inactivated Sendai virus strain Tianjin (UV-Tianjin) has been proved to have antitumor effects in many kinds of tumor cells. Here, we investigated the anticancer properties of UV-Tianjin on human osteosarcoma (HOS) cells and the underlying molecular mechanism. Apoptosis, intracellular reactive oxygen species (ROS) levels and mitochondrial membrane potential were determined by flow cytometry analysis. The expression levels of apoptosis-related proteins were tested by Western blotting. The results showed that UV-Tianjin concentration-dependently induced apoptosis in HOS cells. UV-Tianjin-induced apoptosis was mediated by the mitochondrial pathway, which was confirmed by mitochondrial dysfunction, downregulation of B-cell lymphoma 2 (Bcl-2), B-cell lymphoma-xL (Bcl-xL) and myeloid cell leukemia-1 (Mcl-1), upregulation of $\mathrm{Bcl}-2$-associated $\mathrm{X}$ protein (Bax) and Bcl-2 homologous antagonist/killer (Bak), as well as the cleavage of caspase- 9 and caspase-3. Further analysis showed that UV-Tianjin augmented the phosphorylation of c-Jun $\mathrm{N}$-terminal kinase, the extracellular-regulated kinase and p38, the major components of mitogen-activated protein kinase (MAPK) pathways, as well as the generation of ROS. Moreover, UV-Tianjin-induced apoptosis was remarkably attenuated by MAPK inhibitors and ROS inhibitor. Taken together, our results indicated that UV-Tianjin exerts antitumor effects by inducing mitochondria-dependent apoptosis involving ROS generation and MAPK pathway in human osteosarcoma HOS cells.
\end{abstract}

Key words: Apoptosis - Human osteosarcoma cells - Mitogen-activated protein kinase pathway - Reactive oxygen species - Sendai virus strain Tianjin

\section{Introduction}

Osteosarcoma (OS) is one of the most common primary malignant bone tumors in children and adolescents, and is characterized by poor prognosis and high mortality (Li et al. 2014). The current treatment options for patients with primary OS include neoadjuvant chemotherapy and surgical removal. However, side effects and drug resistance has limited the clinical application of chemotherapeutic agents (Li et al. 2015; Rhea and Oliveira 2018). Therefore, new

\footnotetext{
* These authors contributed equally to this work.

Correspondence to: Liying Shi, Department of Pathogenic Biology, School of Basic Medical Sciences, Tianjin Medical University, No.22, Qixiangtai Road, Heping District, Tianjin 300070, China E-mail: sly.good@126.com shiliying@tmu.edu.cn
}

drugs with better tumor selectivity and fewer side effects are urgently needed.

Over the past decade, cancer-specific oncolytic viruses have received substantial attention for their potential as novel anticancer drugs (Ledford 2015; Varela-Guruceaga et al. 2018). Sendai virus $(\mathrm{SeV})$ is a prototypic member of the family Paramyxoviridae. It has been demonstrated that ultraviolet (UV)-inactivated $\mathrm{SeV}$ may exert anticancer effects by inducing cancer-selective apoptosis, autophage and anticancer immune responses (Kurooka and Kaneda 2007; Zhang et al. 2015; Li et al. 2017). However, the molecular mechanisms underlying its anti-tumor effects have not been fully elucidated.

Sendai virus strain Tianjin was isolated from the lungs of marmosets in 1999. Phylogenetic analysis of complete genome has confirmed that Tianjin strain belonged to Paramyxoviridae and was a new genotype of $\mathrm{SeV}$ (Shi et al. 2008). Our previous studies have demonstrated that UV-inactivated 
Tianjin strain (UV-Tianjin) may induce apoptosis or autophagy of certain cancer cell lines (Chen et al. 2014; Shi et al. 2015; Chen et al. 2016; Han et al. 2019). Whether UV-Tianjin elicits antitumor effect on osteosarcoma HOS cells through apoptosis or autophagy remains unknown.

Apoptosis induction is one of the main mechanisms of most anti-tumor drugs against cancer (Xiang et al. 2016; Dai et al. 2018). It is well known that there are two main signaling pathways to control apoptosis, the mitochondria-dependent pathway and death receptor-dependent pathway (Daniel 2000). In addition, many studies report mitogen-activated protein kinase (MAPK) pathways and reactive oxygen species (ROS) are involved in virus-induced apoptosis (Yang et al. 2010; Olavarría et al. 2015; Qian et al. 2018; Verma et al. 2018). MAPKs consist of growth factor-regulated extracellular signal-related kinases (ERKs), and the stress-activated MAPKs, c-jun NH2-terminal kinases (JNKs) and p38 MAPKs. MAPK pathways are known to be influenced not only by receptor ligand interactions, but also by different stressors placed on the cell. One type of stress that induces potential activation of MAPK pathways is the oxidative stress caused by ROS. Generally, increased ROS production in a cell leads to the activation of ERKs, JNKs, or p38 MAPKs (Son et al. 2011).

In the present study, we prepared UV-inactivated Tianjin strain and determine the antitumor effect and mechanism of UV-Tianjin on human osteosarcoma HOS cells for the first time.

\section{Materials and Methods}

\section{Reagents and antibodies}

Dulbecco's Modified Eagle Medium (DMEM), DMEM/F12, fetal bovine serum (FBS), phosphate buffer saline (PBS) and $0.25 \%$ trypsin were obtained from Gibco BRL, Life Technologies (Grand Island, NY, USA). 3-(4,5-dimethylthiazol-2-yl)-2,5-diphenyl tetrazolium bromide (MTT), $\mathrm{N}$-acetyl-L-cysteine (NAC), caspase-9 specific inhibitor (z-LEHD-fmk), Hoechst 33342 assay kit, annexin V-FITC/ propidium iodide (PI) apoptosis detection kit, mitochondrial membrane potential (MMP) assay kit, and ROS assay kit were purchased from KeyGen Biotech (Nanjing, China). SP600125, SB203580, and PD98059 were purchased from Beyotime (Nanjing, China). Primary antibodies against JNK, p-JNK, p38, p-p38, procaspase-3, Bcl-2 homologous antagonist/killer (Bak), B-cell lymphoma-xL (Bcl-xL), myeloid cell leukemia-1 (Mcl-1) and secondly antibodies were provided by Cell Signaling Technology, Inc. (Danvers, MA, USA). Primary antibodies against B-cell lymphoma 2 (Bcl-2), Bcl2-associated X protein (Bax), cytochrome c (Cyt c), procaspase-9, extracellular signal-regulated kinase (ERK), p-ERK and $\beta$-actin were obtained from Santa Cruz Biotechnology,
Inc. (Dallas, TX, USA). Polyvinylidene difluoride (PVDF) membranes and enhanced chemiluminescence (ECL) detection kit were purchased from Millipore (Billerica, MA, USA).

\section{Cell and viruses}

The human osteosarcoma cell line HOS and human osteoblasts hFOB1.19 were obtained from Cell Bank of Shanghai Institute of Biochemistry and Cell Biology, Chinese Academy of Sciences (Shanghai, China). Cells were cultured in DMEM or DMEM/F12 supplemented with 10\% FBS in a humidified atmosphere containing $5 \% \mathrm{CO}_{2}$. Sendai virus strain Tianjin (GenBank: EF679198.1) was propagated in 9 to 11-day-old specific pathogen free embryonated chicken eggs after inoculation through allantoic route. Inactivation of viral infectivity was achieved by UV irradiation $(99 \mathrm{~mJ} /$ $\mathrm{cm}^{2}$ ), as previously described (Kaneda et al. 2002).

\section{Cell viability assay}

Cell viability was determined by using MTT assay. In brief, cells were seeded onto 96-well plates at a density of $1 \times 10^{4}$ cells/well and incubated for $24 \mathrm{~h}$. Next, cells were treated with various concentration of UV-Tianjin [multiplicity of infection (MOI): 50-800]. After incubating for $24 \mathrm{~h}, 10 \mu \mathrm{l}$ of MTT reagent $(5 \mathrm{mg} / \mathrm{ml}$ ) was added to each well and followed by incubation at $37^{\circ} \mathrm{C}$ for $4 \mathrm{~h}$ in the dark. At the end of the incubation period, the medium was removed and DMSO solution $(100 \mu \mathrm{l})$ was added to each well. The optical density value of each well was measured using a micro-plate reader (Bio-Rad, Hercules, CA, USA) at a wavelength of $570 \mathrm{~nm}$.

\section{Hoechst staining}

HOS cells were seeded in 6-well plates at $2.0 \times 10^{5}$ cells/well. After reaching confluence, cells were treated with UV-Tianjin (MOI: 100, 200 and 400) for $24 \mathrm{~h}$. Then the culture medium was removed and the cells were stained with Hoechst 33342 at $37^{\circ} \mathrm{C}$ for $15 \mathrm{~min}$ under dark condition. The cells were washed with PBS and observed with a fluorescence microscope (Nikon Eclipse E600, Tokyo, Japan).

\section{Annexin V-FITC/PI analysis for cell apoptosis}

The apoptosis induced by UV-Tianjin was measured with annexin V/PI double staining assay, according to the manufacturer's protocol. Briefly, HOS cells were seeded in a 6-well plate and then treated with UV-Tianjin (MOI 100, 200 and 400) for $24 \mathrm{~h}$. At the end of the treatment, cells were digested, washed and then incubated with annexin V and PI at room temperature for $10 \mathrm{~min}$ in the dark. The stained cells were analyzed with a flow cytometry (Becton \& Dickinson, Franklin Lakes, NJ, USA). 


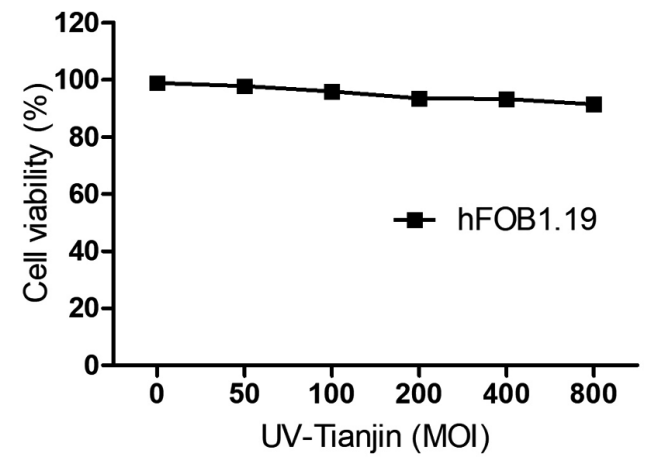

Figure 1. Effect of UV-Tianjin on the viability of hFOB 1.19 osteoblasts. Cells in a 96-well plate were treated with 50, 100, 200, 400 and $800 \mathrm{MOI}$ of UV-Tianjin for $24 \mathrm{~h}$. Cell viability was detected by MTT assay. The data are shown as mean \pm SEM of three independent experiments. MOI, multiplicity of infection; UV-Tianjin, ultraviolet-inactivated Sendai virus strain Tianjin.

\section{Mitochondrial membrane potential $\left(\Delta \psi_{m}\right)$ analysis}

HOS cells were cultured in 6-well plates overnight and then exposed to UV-Tianjin (MOI 100, 200 and 400) for $24 \mathrm{~h}$. Cells were harvested, washed and incubated with JC-1 dye for $30 \mathrm{~min}$ at $37^{\circ} \mathrm{C}$, and then rinsed with JC-1 staining buffer twice. The $\Delta \psi_{\mathrm{m}}$ of HOS cells were detected by flow cytometry.

\section{Western blot analysis}

After treated with different doses of UV-Tianjin (MOI: 100, 200 and 400) for $24 \mathrm{~h}$, the cells were collected and lysed in radioimmunoprecipitation assay lysis buffer for $30 \mathrm{~min}$ on ice. After centrifugation, the concentration of protein was determined using a bicinchoninic acid protein assay kit. The samples were loaded to $10 \%$ sodium dodecyl sulphate-polyacrylamide gel electrophoresis gels and then transferred to a PVDF membrane. The membranes were blocked for $1 \mathrm{~h}$ at room temperature with $5 \%$ non-fat milk in Tris-buffered saline-Tween (TBST) and then incubated with the primary antibodies at $4^{\circ} \mathrm{C}$ overnight. After washing with TBST, membranes were incubated with a secondary antibody for $2 \mathrm{~h}$. Protein bands were visualized using an ECL kit.

\section{Measurement of reactive oxygen species}

Intracellular ROS was detected with the fluorescent probe DCFH-DA according to the manufacturer's protocol. Briefly, following the treatment with UV-Tianjin (MOI: 100, 200 and $400)$ for $24 \mathrm{~h}$, cells were incubated with DCFH-DA $(10 \mu \mathrm{M})$ for $20 \mathrm{~min}$ at $37^{\circ} \mathrm{C}$ in the dark and then washed three times with PBS. DCF fluorescence intensity was measured with flow cytometry.

\section{Statistical analysis}

All experiments were performed at least three times and data were expressed as mean \pm standard error of mean (SEM). Statistical analysis was performed by SPSS 19.0 software (SPSS Inc., Chicago, IL, USA). Comparisons between groups were made using one-way analysis of variance followed by Tukey's test. Results were considered statistically significant when the $p$ value was less than 0.05 .

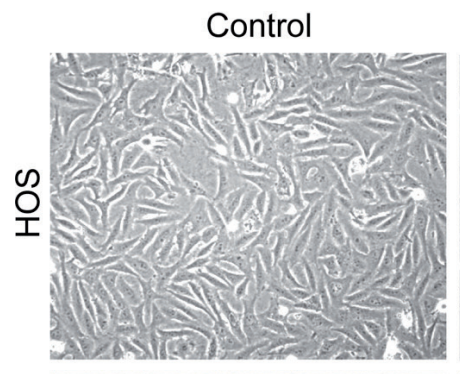

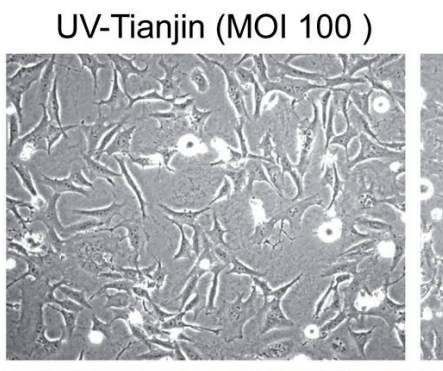

UV-Tianjin (MOI 200)
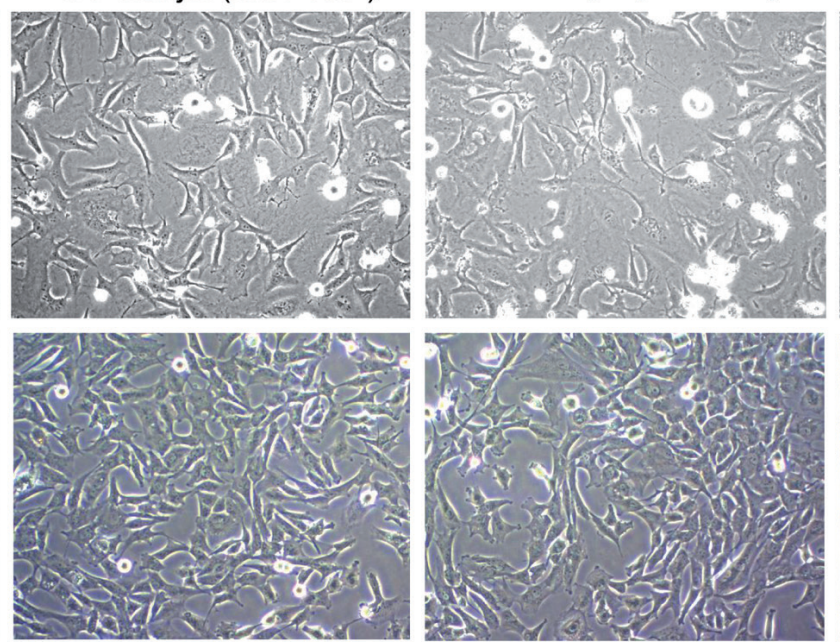

UV-Tianjin (MOI 400)

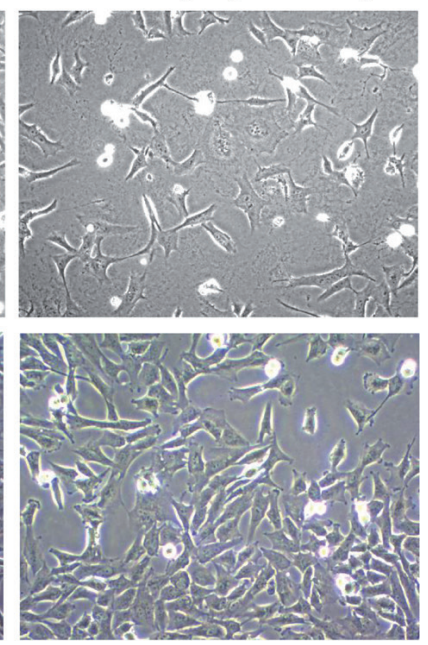

Figure 2. Effect of UV-Tianjin on the morphology of HOS cells and hFOB 1.19 osteoblasts. The morphological changes of HOS cells or hFOB 1.19 osteoblasts were examined by an inverted microscopy ( $\times 100)$ after $24 \mathrm{~h}$ of treatment with UV-Tianjin (MOI 100, 200 and 400). For abbreviations, see Fig. 1. 


\section{Results}

UV-Tianjin had no significant effect on the viability of human osteoblasts hFOB1.19

Our previous study has shown that treatment with UVTianjin for $24 \mathrm{~h}$ resulted in a significant dose-dependent decrease in cell viability of HOS cells by MTT assay (Han et al. 2019). In the present study, the effect of UV-Tianjin on the viability of human osteoblasts hFOB1.19 was examined. As shown in Fig. 1, viability of human osteoblasts hFOB1.19 was not significantly affected by UV-Tianjin treatment, indicating a strong selective effect of UV-Tianjin towards cancer cells. On the other hand, microscopic observation showed that UVTianjin induced significant cellular morphological changes in HOS cells, but not in hFOB1.19 osteoblasts (Fig. 2).

\section{UV-Tianjin induced apoptosis in HOS cells}

Firstly, the effect of UV-Tianjin on apoptosis of HOS cells was examined using Hoechst 33342 staining. We observed that, in a dose-dependent manner, the chromatin became condensed and marginalized following the treatment of UV-Tianjin (Fig. 3A). Subsequently, apoptosis was detected
A

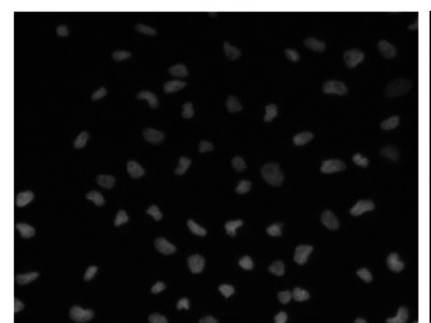

B
UV-Tianjin (MOI 100)

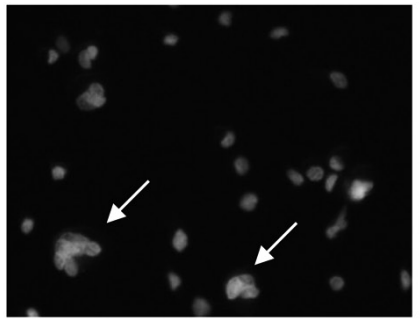

UV-Tianjin (MOI 200)

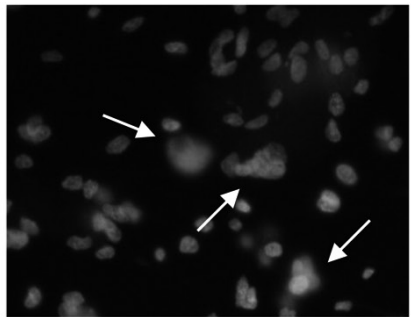

UV-Tianjin (MOI 400)

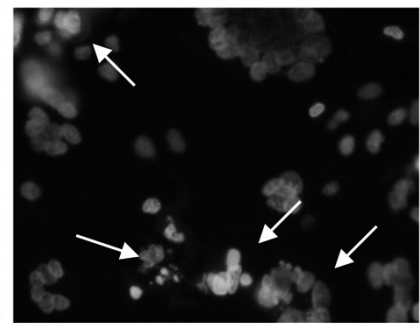

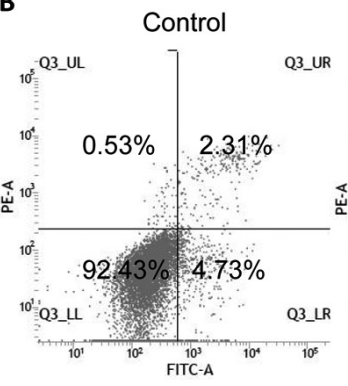

D

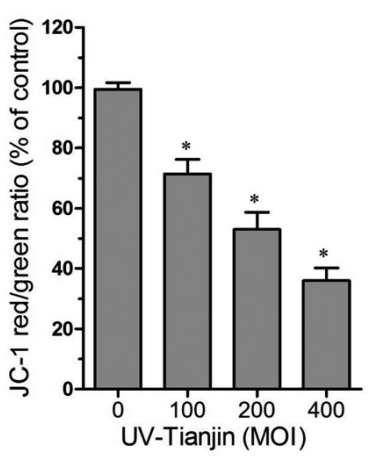

UV-Tianjin (MOI 100)

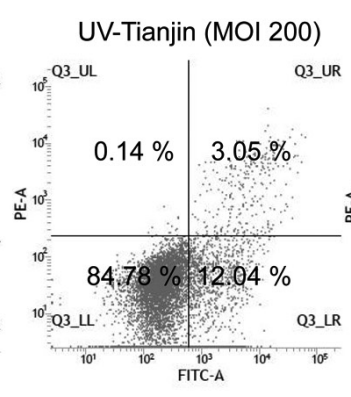

$\mathbf{E}$

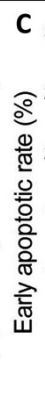

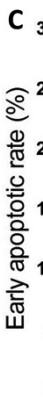

$\mathbf{F}$ UV-Tianjin (MOI 400)

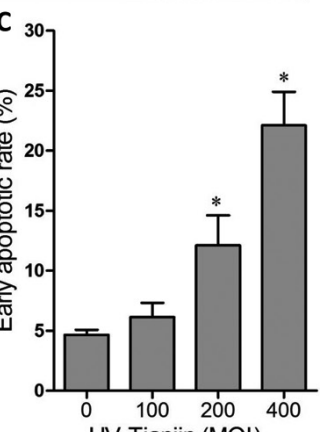

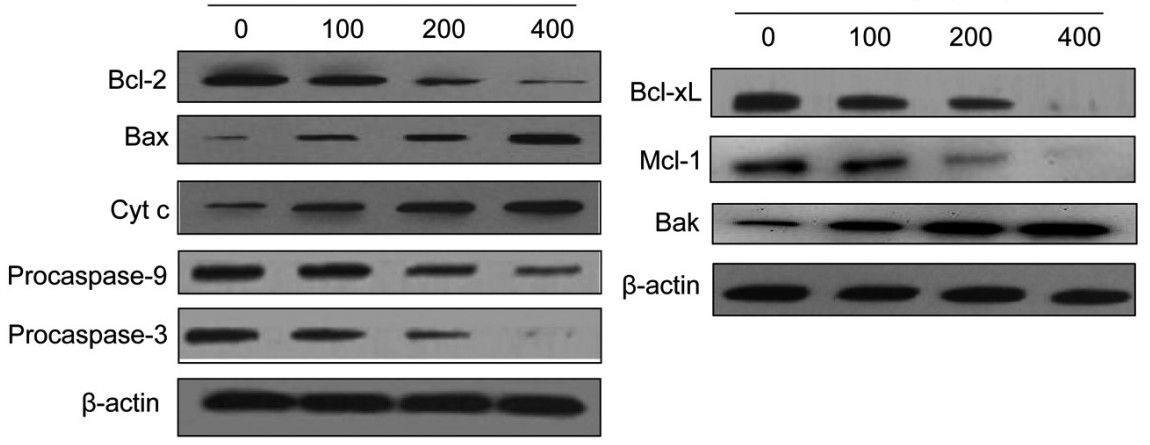

Figure 3. UV-Tianjin triggered apoptosis through the mitochondrial pathway in HOS cells. HOS cells were treated with UV-Tianjin at the indicated concentrations for $24 \mathrm{~h}$. A. Nuclear condensation and fragmentation are detected by Hoechst 33342 staining $(\times 200)$. Arrows indicate condensed chromatin and nuclear fragmentation. B. HOS cells were stained with annexin V-FITC/PI and determined by flow cytometry. Representative images were presented. C. The histogram illustrates the percentages of early apoptotic cells. D. The $\Delta \psi \mathrm{m}$ was assessed by flow cytometry using JC-1 staining. Quantitative analysis in histogram was presented. E. and F.. Western blotting was used to analyze the expression of Bcl-2, Bax, Cyt c, procaspase-9, procaspase-3, Bcl-xL, Mcl-1 and Bak proteins. Results are expressed as the mean \pm SEM from three independent experiments. ${ }^{\star} p<0.05$ vs. untreated control. Bak, Bcl-2 homologous antagonist/killer; Bax, Bcl-2-associated X protein; Bcl-2, B-cell lymphoma 2; Bcl-xL, B-cell lymphoma-xL; Cyt c, cytochrome c; Mcl-1, myeloid cell leukemia-1. For more abbreviations, see Fig. 1. 

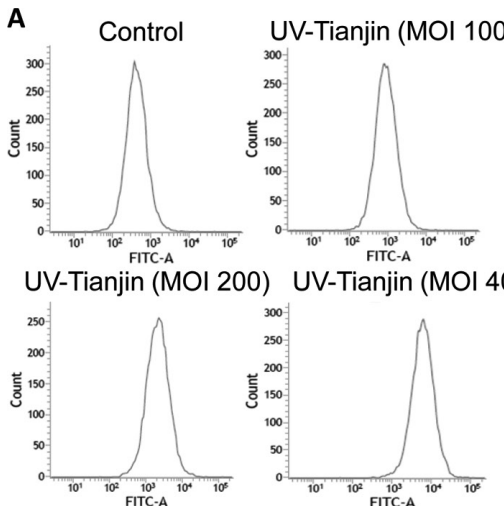

C

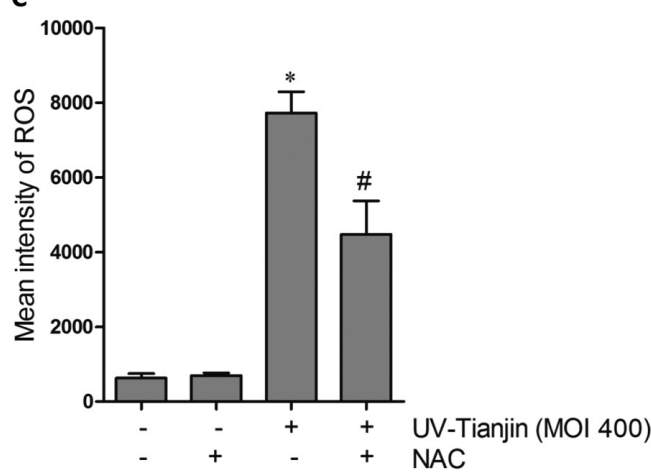

E

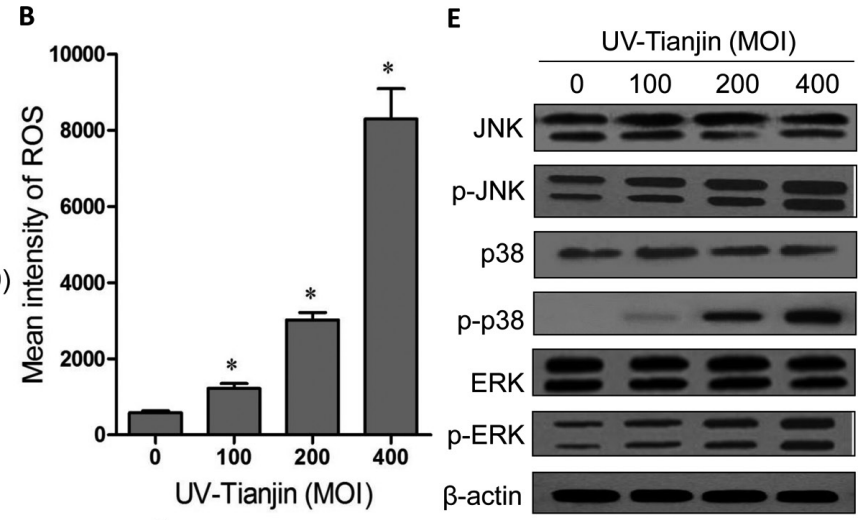

D

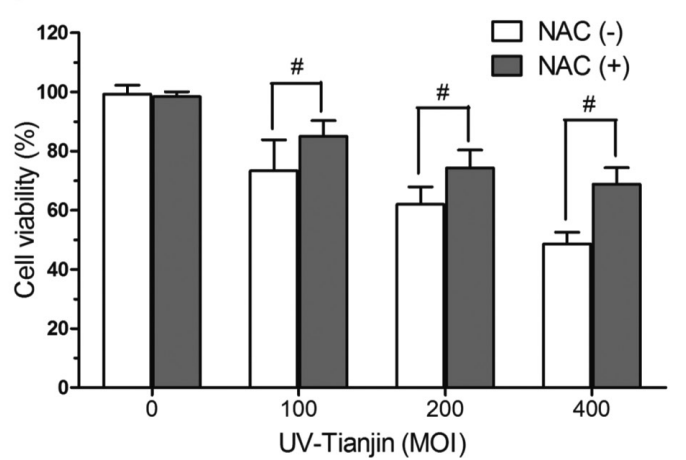

Figure 4. Effects of UV-Tianjin on intracellular ROS and MAPK pathway in HOS cells. HOS cells were treated with UV-Tianjin at the indicated concentrations for $24 \mathrm{~h}$. A. The level of ROS was determined by flow cytometry. Representative images were presented. B. Quantitative analysis of ROS generation was shown in histogram. C. HOS cells were pretreated with or without NAC (5 mM) for $1 \mathrm{~h}$, and then followed by UV-Tianjin at a MOI of 400 for $24 \mathrm{~h}$. The ROS level was analyzed by flow cytometry. Quantitative analysis was shown in histogram. D. HOS cells were pretreated with or without NAC (5 mM) for $1 \mathrm{~h}$, and then followed by UV-Tianjin (MOI 100, 200, 400) for $24 \mathrm{~h}$. Cell viability was assessed by MTT assay. E. The expression of JNK, p-JNK, ERK, p-ERK, p38 and p-p38 were measured by Western blotting. Results are expressed as the mean \pm SEM from three independent experiments. ${ }^{*} p<0.05$ vs. untreated control. ${ }^{\#} p<0.05$ vs. the single UV-Tianjin treatment groups. ERK, extracellular signal-regulated kinase; JNK, c-Jun N-terminal kinases; NAC, $\mathrm{N}$-acetylcysteine; ROS, reactive oxygen species. For more abbreviations, see Fig. 3.

by annexin V/PI double-staining using flow cytometry. UVTianjin treatment induced a dose-dependent increase in the percentage of early apoptotic cells (Fig. 3B and 3C). These results clearly indicate that UV-Tianjin provoked apoptosis in HOS cells.

\section{UV-Tianjin induced apoptosis through mitochondrial pathway in HOS cells}

Next, we studied the effect of UV-Tianjin on mitochondria. As shown in Fig. 3D, a loss of $\Delta \psi_{\mathrm{m}}$ was indicated by a decreased in the ratio of red fluorescence to green fluorescence following UV-Tianjin treatment. Furthermore, we investigated the expression of proteins associated with mitochondria-mediated apoptosis. As shown in Fig. $3 \mathrm{E}$ and 3F, UV-Tianjin treatments dose-dependently decreased the expression of $\mathrm{Bcl}-2, \mathrm{Bcl}-\mathrm{xL}, \mathrm{Mcl}-1$, procaspase- 9 and -3 and increased the levels of Bax, Bak and
Cyt $c$ in HOS cells. To further confirm the findings, we investigated the role of caspase-9, a marker of the mitochondrial pathway, using the caspase- 9 specific inhibitor z-LEHD-fmk. Expectedly, flow cytometry analysis showed that $\mathrm{z}$-LEHD-fmk significantly inhibited the apoptosis induced by UV-Tianjin (Fig. 5A and 5B). These results clearly demonstrated that UV-Tianjin-induced apoptosis involves the mitochondrial pathway.

\section{UV-Tianjin enhanced ROS levels in HOS cells}

In order to explore the role of ROS in UV-Tianjin-mediated apoptosis, we examined the intracellular production of ROS. As shown in Fig. 4A and B, intracellular ROS production was significantly increased by UV-Tianjin, compared to control. To further confirm the involvement of ROS in UV-Tianjininduced apoptosis, HOS cells were exposed to NAC ( $5 \mathrm{mM})$, the ROS scavenger, for $1 \mathrm{~h}$ and then treated with UV-Tianjin 
A

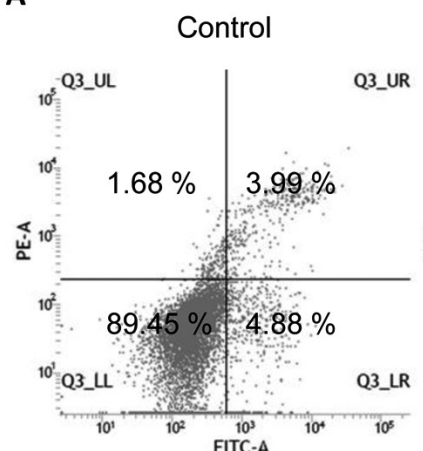

UV-Tianjin (MOI 400)
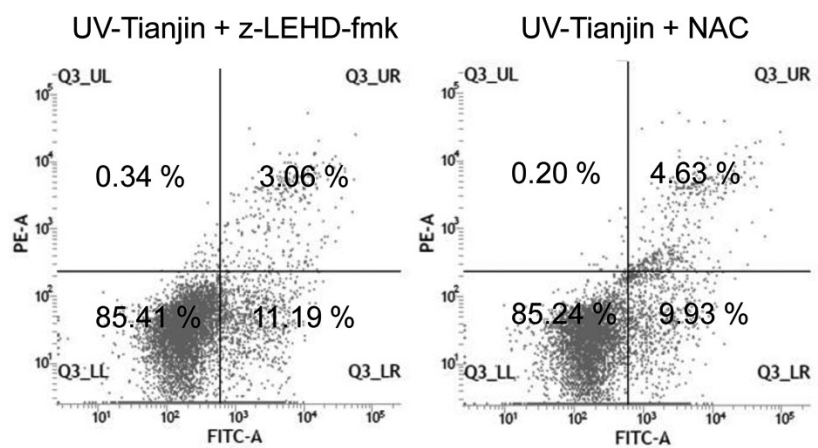

B

D

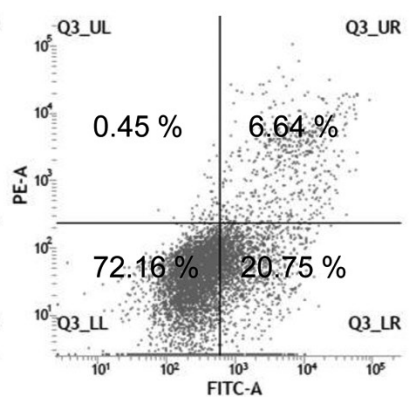

\section{E}
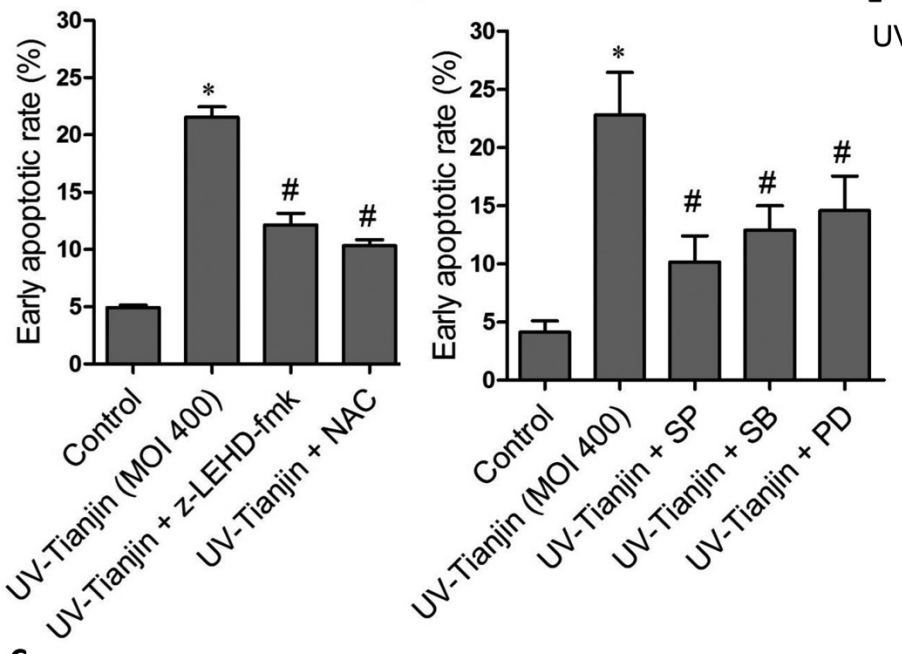

UV-Tianjin (MOI 400)
SP $(20 \mu \mathrm{M})$
SB $(20 \mu \mathrm{M})$
PD $(5 \mu \mathrm{M})$
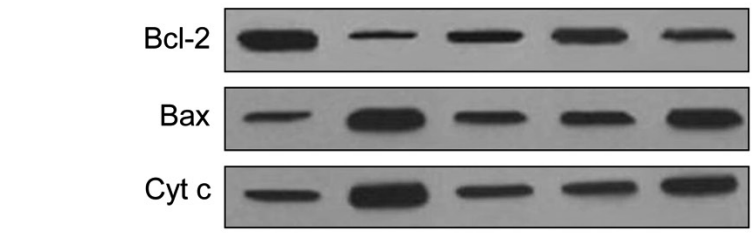

Procaspase 9

Procaspase 3

$\beta$-actin

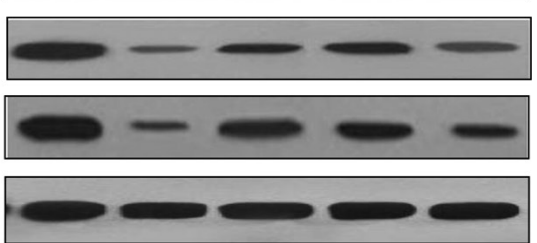

C
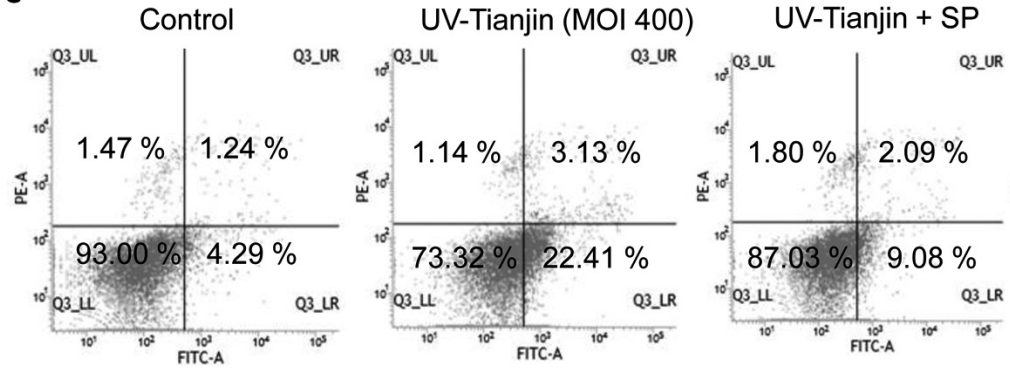

UV-Tianjin + SB

UV-Tianjin + PD
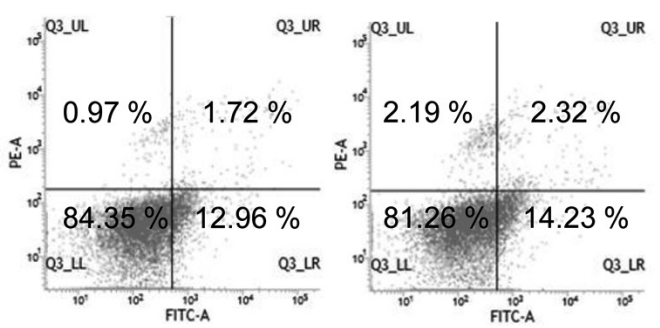

Figure 5. Inhibition of caspase, ROS production and MAPK pathway attenuated UV-Tianjin-induced apoptosis in HOS cells. HOS cells were preincubated with caspase-9 specific inhibitor z-LEHD-fmk $(20 \mu \mathrm{M})$, ROS inhibitor NAC $(5 \mathrm{mM})$, JNK inhibitor SP $(20 \mu \mathrm{M})$, ERK inhibitor PD $(5 \mu \mathrm{M})$ or p38 inhibitor SB $(20 \mu \mathrm{M})$ for $1 \mathrm{~h}$, and then treated with UV-Tianjin (MOI 400) for $24 \mathrm{~h}$. A-D. Induction of apoptosis was assessed by flow cytometry using annexin V and PI staining. Quantitative analysis of early apoptosis rate was presented in histograms. E. The expressions of apoptosis-related proteins were detected by Western blotting. Representative images were presented. Results expressed as mean \pm SEM of three independent assays. ${ }^{\star} p<0.05 v$ s. control. ${ }^{\#} p<0.05 v s$. the single UV-Tianjin treatment groups. PD, PD98059; SB, SB203580; SP, SP600125. For more abbreviations, see Fig. 4.

for $24 \mathrm{~h}$. As was expected, addition of NAC significantly decreased the level of ROS (Fig. 4C) and attenuated UVTianjin-induced cell viability loss (Fig. 4D) and apoptosis to a certain extent (Fig. 5A and 5B). These results suggest that increased ROS production in HOS cells plays an important role in UV-Tianjin-induced apoptosis.
UV-Tianjin induced apoptosis through MAPK pathway in HOS cells

To further elucidate the underlying mechanism of apoptosis induced by UV-Tianjin, we evaluated the activation of MAPK signaling pathway, which play critical roles in 
apoptosis regulation. As shown in Fig. 4E, the phosphorylation of JNK, ERK and p38, the major components of MAPK pathway, was increased in a dose-dependent manner in HOS cells treated with UV-Tianjin. To further determine the role of MAPKs in UV-Tianjin-induced apoptosis in HOS cells, JNK inhibitor SP600125 $(20 \mu \mathrm{M})$, ERK inhibitor PD98059 $(5 \mu \mathrm{M})$ and p38 inhibitor SB203580 $(20 \mu \mathrm{M})$ were used to pretreat HOS cells before the treatment of UV-Tianjin. As shown in Fig. 5C, D and E, MAPK inhibitors could significantly reduce the apoptosis of HOS cells and attenuated the expression of apoptosis-related proteins trigged by UV-Tianjin. Overall, these results indicated that MAPK signaling pathway might mediate UV-Tianjininduced apoptosis in HOS cells.

\section{Discussion}

Recently, SeV has been identified as a potential anticancer agent in many studies (Kurooka and Kaneda 2007; Zhang et al. 2015; Gao et al. 2016; Li et al. 2017). However, the molecular mechanisms for the anticancer activity of SeV are poorly understood. Our latest study showed that UVTianjin, a new genotype of SeV, significantly suppressed the proliferation of HOS cells in a dose-dependent manner (Han et al. 2019). In the present study, we found that UVTianjin had no significant effect on the viability of human osteoblasts hFOB1.19, indicating a strong selective effect of UV-Tianjin towards cancer cells. Moreover, the present study also showed that inhibition of proliferation of HOS cells was mostly related to cell apoptosis, as evidenced by Hoechst staining and flow cytometry analysis, suggesting that the anticancer effect of UV-Tianjin on HOS cells was mediated through the induction of apoptosis.

The intrinsic, mitochondrial pathway is activated by intracellular signals generated in response to cellular stress. It is regulated by Bcl-2 family proteins, including the antiapoptotic factor (Bcl-2, Bcl-xL, Mcl-1) and the pro-apoptotic factor (Bax, Bak) (D'Orsi et al. 2017). An increase of Bax/ Bcl-2 ratio may induce a loss in $\Delta \psi_{\mathrm{m}}$, release of Cyt $\mathrm{c}$ into the cytoplasm, and subsequent activation of caspase, which eventually leads to occurrence of cell apoptosis (Matsuyama and Reed 2000). In our results, UV-Tianjin decreased the ratio of red/green fluorescence intensity, indicating a decreased $\Delta \psi_{\mathrm{m}}$ and depolarization of the mitochondrial membrane. In addition, our results also demonstrated that UV-Tianjin decreased the levels of Bcl-2, Bcl-xL, Mcl-1, procaspase-9 and procaspase- 3 and increased the levels of Bax, Bak, and Cyt c in a dose-dependent manner. Moreover, the caspase- 9 specific inhibitor z-LEHD-fmk significantly attenuated UV-Tianjin-induced apoptosis. All these evidences suggest that the mitochondrial pathway is involved in UV-Tianjininduced apoptosis in HOS cells.
Many chemotherapeutic agents exert their anticancer effects by inducing the generation of ROS (Park et al. 2015; Wang et al. 2016). In the present study, the production of intracellular ROS increased remarkably in HOS cells treated with UV-Tianjin, while inhibition of ROS production by the antioxidant NAC attenuated UV-Tianjin-induced cell viability loss and apoptosis, suggesting that UV-Tianjininduced apoptosis in HOS cells was closely associated with the production of ROS, which may act as upstream signaling molecules to initiate mitochondria-mediated cell apoptosis (Wang 2001).

As one of the most important signal transduction systems of organism, MAPK pathways participate in many cellular physiological functions, including cellular proliferation and apoptosis (Sun et al. 2015). Our results demonstrated that UV-Tianjin increased the levels of phosphorylated JNK, p38 and ERK in a dose-dependent manner in HOS cells. Moreover, UV-Tianjin-induced apoptosis was remarkably attenuated by JNK inhibitor SP600125, ERK inhibitor PD98059 and p38 inhibitor SB203580, suggesting that MAPK pathways were involved in UV-Tianjin-induced apoptosis in HOS cells.

In summary, these results demonstrated that UV-Tianjin induced apoptotic cell death in HOS cells through the intrinsic mitochondrial pathways via triggering ROS generation and activating MAPK pathways, suggesting that UV-Tianjin might be a potential candidate for the treatment of human osteosarcoma. This study provides an insight into the molecular mechanisms of SeV-induced apoptosis in human osteosarcoma cells.

Acknowledgments. The present study was supported by National Natural Science Foundation of China (No.81172168).

Conflict of interest. The authors have no conflicts of interest to declare.

\section{References}

Chen J, Han H, Chen M, Xu XZ, Wang B, Shi LY (2014): Inactivated Sendai virus strain Tianjin induces apoptosis in human breast cancer MDA-MB-231 cells. Asian Pac. J. Cancer Prev. $15,5023-5028$ https://doi.org/10.7314/APJCP.2014.15.12.5023

Chen J, Han H, Wang B, Shi L (2016): Inactivated Tianjin strain, a novel genotype of Sendai virus, induces apoptosis in HeLa, NCI-H446 and Hep3B cells. Oncol. Lett. 12, 49-56 https://doi.org/10.3847/0004-637X/825/1/49

Dai X, Li RZ, Jiang ZB, Wei CL, Luo LX, Yao XJ, Li GP, Leung EL (2018): honokiol inhibits proliferation, invasion and induces apoptosis through targeting lyn kinase in human lung adenocarcinoma cells. Front. Pharmaco. 19, 558 https://doi.org/10.3389/fphar.2018.00558

Daniel PT (2000): Dissecting the pathways to death. Leukemia 14, 2035-2044 
https://doi.org/10.1038/sj.leu.2401940

D`Orsi B, Mateyka J, Prehn JHM (2017): Control of mitochondrial physiology and cell death by the Bcl-2 family proteins Bax and Bok. Neurochem. Int. 109, 162-170 https://doi.org/10.1016/j.neuint.2017.03.010

Gao H, Li LY, Zhang M, Zhang Q (2016): Inactivated Sendai virus induces apoptosis mediated by reactive oxygen species in murine melanoma cells. Biomed. Environ. Sci. 29, 877-884

Han Z, Li Q, Sun S, Zhao W, Shi L (2019): Inactivated Sendai virus strain Tianjin induces apoptosis and autophagy through reactive oxygen species production in osteosarcoma MG-63 cells. J. Cell Physiol. 234, 4179-4190 https://doi.org/10.1002/jcp.27176

Kaneda Y, Nakajima T, Nishikawa T, Yamamoto S, Ikegami H, Suzuki N, Nakamura H, Morishita R, Kotani H (2002): Hemagglutinating virus of Japan (HVJ) envelope vector as a versatile gene delivery system. Mol. Ther. 6, 219-226 https://doi.org/10.1006/mthe.2002.0647

Kurooka M, Kaneda Y (2007): Inactivated Sendai virus particles eradicate tumors by inducing immune responses through blocking regulatory T cells. Cancer Res. 67, 227-236 https://doi.org/10.1158/0008-5472.CAN-06-1615

Ledford H (2015): Cancer-fighting viruses win approval. Nature 526, 622-623 https://doi.org/10.1038/526622a

Li Y, Huang Y, Lv Y, Meng G, Guo QN (2014): Epigenetic regulation of the pro-apoptosis gene TSSC 3 in human osteosarcoma cells. Biomed. Pharmacother. 68, 45-50 https://doi.org/10.1016/j.biopha.2013.10.006

Li S, Sun W, Wang H, Zuo D, Hua Y, Cai Z (2015): Research progress on the multidrug resistance mechanisms of osteosarcoma chemotherapy and reversal. Tumor Biol. 36, 1329-1338 https://doi.org/10.1007/s13277-015-3181-0

Li S, Nishikawa T, Kaneda Y (2017): Inactivated Sendai virus particle upregulates cancer cell expression of intercellular adhesion molecule- 1 and enhances natural killer cell sensitivity on cancer cells. Cancer Sci. 108, 2333-2341

https://doi.org/10.1111/cas.13408

Matsuyama S, Reed JC (2000): Mitochondria-dependent apoptosis and cellular pH regulation. Cell Death Differ. 7, 1155-1165 https://doi.org/10.1038/sj.cdd.4400779

Olavarría VH, Recabarren P, Fredericksen F, Villalba M, Yáñez A (2015): ISAV infection promotes apoptosis of SHK-1 cells through a ROS/p38 MAPK/Bad signaling pathway. Mol. Immunol. 64, 1-8 https://doi.org/10.1016/j.molimm.2014.10.016

Park SW, Kim JE, Oh SM, Cha WJ, Hah JH, Sung MW (2015): Anticancer effects of anandamide on head and neck squamous cell carcinoma cells via the production of receptor-independent reactive oxygen species. Head Neck 37, 1187-1192 https://doi.org/10.1002/hed.23727

Qian M, Tan HM, Yu N, Wang T, Zhang Q (2018): Inactivated sendai virus Induces ROS-dependent apoptosis and autophagy in human prostate cancer cells. Biomed. Environ. Sci. 31, 280-289

Rhea IB, Oliveira GH (2018): Cardiotoxicity of Novel Targeted Chemotherapeutic Agents. Curr. Treat. Options Cardiovasc. Med. 20, 53 https://doi.org/10.1007/s11936-018-0649-4

Shi LY, Li M, Yuan LJ, Wang Q, Li XM (2008): A new paramyxovirus, Tianjin strain, isolated from common cotton-eared marmoset: Genome characterization and structural protein sequences analysis. Arch. Virol. 153, 1715-1723 https://doi.org/10.1007/s00705-008-0184-9

Shi LY, Han Z, Li XX, Li M, Han H, Chen J, Zang S (2015): Inactivated Sendai virus strain Tianjin induces apoptosis in breast cancer MCF-7 cells by promoting caspase activation and Fas/ FasL expression. Cancer Biother. Radiopharm. 30, 33-40 https://doi.org/10.1089/cbr.2014.1704

Son Y, Cheong YK, Kim NH, Chung HT, Kang DG, Pae HO (2011): Mitogen-activated protein kinases and reactive oxygen species: how can ROS activate MAPK pathways? J. Signal Transduct. 2011, 792639 https://doi.org/10.1155/2011/792639

Sun Y, Liu WZ, Liu T, Feng X, Yang N, Zhou HF (2015): Signaling pathway of MAPK/ERK in cell proliferation, differentiation, migration, senescence and apoptosis. J. Recept Signal Transduct. Res. 35, 600-604 https://doi.org/10.3109/10799893.2015.1030412

Varela-Guruceaga M, Tejada-Solís S, García-Moure M, Fueyo J, Gomez-Manzano C, Patiño-García A, Alonso MM (2018): Oncolytic viruses as therapeutic tools for pediatric brain tumors. Cancers (Basel) 10, E226 https://doi.org/10.3390/cancers10070226

Verma AK, Ghosh S, Basu A (2018): chandipura virus induced neuronal apoptosis via calcium signaling mediated oxidative stress. Front. Microbiol. 9, 1489 https://doi.org/10.3389/fmicb.2018.01489

Wang XD (2001): The expanding role of mitochondria in apoptosis. Gene Dev. 15, 2922-2933

Wang R, Ma L, Weng D, Yao J, Liu X, Jin F (2016): Gallic acid induces apoptosis and enhances the anticancer effects of cisplatin in human small cell lung cancer $\mathrm{H} 446$ cell line via the ROS-dependent mitochondrial apoptotic pathway. Oncol. Rep. 35, 3075-3083 https://doi.org/10.3892/or.2016.4690

Xiang M, Su H, Shu G, Wan D, He F, Loaec M, Ding Y, Li J, Dovat S, Yang G, Song C (2016): Amplexicaule A exerts anti-tumor effects by inducing apoptosis in human breast cancer. Oncotarget 7, 18521-18530 https://doi.org/10.18632/oncotarget.7848

Yang TC, Lai CC, Shiu SL, Chuang PH, Tzou BC, Lin YY, Tsai FJ, Lin CW (2010): Japanese encephalitis virus down-regulates thioredoxin and induces ROS-mediated ASK1-ERK/p38 MAPK activation in human promonocyte cells. Microbes Infect. 12, 643-651 https://doi.org/10.1016/j.micinf.2010.04.007

Zhang Q, Zhu H, Xu X, Li L, Tan H, Cai X (2015): Inactivated Sendai virus induces apoptosis and autophagy via the PI3K/Akt/ mTOR/p70S6K pathway in human non-small cell lung cancer cells. Biochem. Biophys. Res. Commun. 465, 64-70 https://doi.org/10.1016/j.bbrc.2015.07.130

Received: January 30, 2019

Final version accepted: April 12, 2019

First published online: June 20, 2019 